\title{
Attenuated Corynebacterium Parvum
}

National Cancer Institute

\section{Source}

National Cancer Institute. Attenuated Corynebacterium Parvum. NCI Thesaurus. Code C780.

A heat-inactivated preparation of Corynebacterium parvum with immunoadjuvant

properties. Therapeutic Corynebacterium parvum may stimulate host antitumor immune responses when added to cancer vaccines. ( $\mathrm{NCl04)}$ 